\title{
The effect of rosuvastatin on incident pneumonia: results from the JUPITER trial
}

\author{
Victor Novack MD PhD, Jean MacFadyen BA, Atul Malhotra MD, Yaniv Almog MD, Robert J. Glynn ScD, \\ Paul M. Ridker MD MPH
}

\begin{abstract}
Background: Evidence from observational studies have raised the possibility that statin treatment reduces the incidence of certain bacterial infections, particularly pneumonia. We analyzed data from a randomized controlled trial of rosuvastatin to examine this hypothesis.
\end{abstract}

Methods: We analyzed data from the randomized, double-blind, placebo-controlled JUPITER trial (Justification for the Use of Statins in Prevention: an Intervention Trial Evaluating Rosuvastatin). In this trial, 17802 healthy participants (men 50 years and older and women 60 and older) with a low-density lipoprotein (LDL) cholesterol level below $130 \mathrm{mg} / \mathrm{dL}$ ( $3.4 \mathrm{mmol} / \mathrm{L})$ and a high-sensitivity C-reactive protein level of $2.0 \mathrm{mg} / \mathrm{L}$ or greater were randomly assigned to receive either rosuvastatin or placebo. We evaluated the incidence of pneumonia on an intention-to-treat basis by reviewing reports of adverse events from the study investigators, who were unaware of the treatment assignments.
Results: Among 17802 trial participants followed for a median of 1.9 years, incident pneumonia was reported as an adverse event in 214 participants in the rosuvastatin group and 257 in the placebo group (hazard ratio [HR] 0.83, 95\% confidence interval [CI] 0.691.00). In analyses restricted to events occurring before a cardiovascular event, pneumonia occurred in 203 participants given rosuvastatin and 250 given placebo (HR 0.81, 95\% Cl 0.670.97). Inclusion of recurrent pneumonia events did not modify this effect (HR $0.81,95 \% \mathrm{Cl}$ 0.67-0.98), nor did adjustment for age, sex, smoking, metabolic syndrome, lipid levels and C-reactive protein level.

Interpretation: Data from this randomized controlled trial support the hypothesis that statin treatment may modestly reduce the incidence of pneumonia.

(ClinicalTrials.gov trial register no. NCT0023968.)
Competing interests: See end of article for competing interests.

This article has been peer reviewed.

Correspondence to:

Dr. Victor Novack,

victorno@clalit.org.il

CMAJ 2011. DOI:10.1503/ cmaj.111017
$\mathrm{R}$ andomized trials of statin treatment have consistently shown reductions in the incidence of cardiovascular events. ${ }^{1}$ In addition to these proven vascular effects, several observational studies have raised the possibility that statins reduce the incidence and severity of certain bacterial infections, ${ }^{2-5}$ particularly pneumonia. ${ }^{6-9}$ Mechanistic support for this hypothesis is provided in part by laboratory evidence that statins, in addition to lowering low-density lipoprotein (LDL) cholesterol levels considerably, have a positive effect on inflammation, apoptosis, antioxidant balance and endothelial function. ${ }^{10}$ However, a common confounder typical of these observational studies relates to the fact that statin treatment may be a nonspecific marker of improved quality of care (healthy user effect). ${ }^{11,12}$ In addition, because infections such as pneumonia are a common complication of myocardial infarction and stroke, any beneficial effect of statin treatment on pneumonia and other infections reported in observational studies may have been due simply to a reduction in these vascular events.

We reviewed data from the recently completed JUPITER trial (Justification for the Use of Statins in Prevention: an Intervention Trial Evaluating Rosuvastatin), a randomized controlled trial involving more than 17000 men and women randomly assigned to receive either rosuvastatin or placebo, to examine the possibility that statins may reduce the incidence of pneumonia.

\section{Methods}

\section{Study population}

The JUPITER trial was a randomized, doubleblind, placebo-controlled multicentre trial conducted at 1315 sites in 26 countries. ${ }^{13}$ Men 50 years of age or older and women 60 years of age or older were eligible for inclusion if they had no history of cardiovascular disease or diabetes and, at the initial screening visit, had an LDL cholesterol level below $130 \mathrm{mg} / \mathrm{dL}(3.4 \mathrm{mmol} / \mathrm{L})$ and a high-sensitivity $\mathrm{C}$-reactive protein level of 
$2.0 \mathrm{mg} / \mathrm{L}$ or greater. ${ }^{13,14}$ Other requirements included a willingness to participate for the duration of the trial, provision of written informed consent and a triglyceride level below $500 \mathrm{mg} / \mathrm{dL}$ $(5.6 \mathrm{mmol} / \mathrm{L})$.

Exclusion criteria included the use of lipidlowering therapy within six weeks before screening, current use of postmenopausal hormone-replacement therapy, cancer within five years before enrolment (except for basal cell or squamous-cell carcinoma of the skin), diabetes and uncontrolled hypertension. Patients were also excluded if they had inflammatory conditions (e.g., severe arthritis, lupus or inflammatory bowel disease) or if they were taking immunosuppressant agents (e.g., cyclosporine, tacrolimus, azathioprine and long-term oral glucocorticoid treatment).

\section{Randomization and follow-up}

Between Mar. 14, 2003, and Dec. 15, 2006, a total of 17802 participants were randomly assigned in a 1:1 ratio to receive either $20 \mathrm{mg}$ of rosuvastatin daily or placebo. Follow-up visits were scheduled to occur at 13 weeks and at 6 , $12,18,24,30,36,42,48,54$ and 60 months after group assignment.

On Mar. 30, 2008, the trial's steering committee accepted the recommendation of the independent data and safety monitoring board to end the trial on the basis of convincing evidence of efficacy with respect to the primary cardiovascular outcome. Participants were asked to continue their blinded treatment and were followed for adverse events, including infections, until they attended a closeout visit, when they learned their treatment assignment. The last closeout visit occurred on Aug. 20, 2008.

\section{Outcome measures}

We evaluated the incidence of infections occurring between treatment assignment and unblinding at the final closeout visit by reviewing reports of adverse events from the study investigators, who were unaware of the treatment assignments. The adverse events were coded by the investigators using MedDRA-11 criteria. (MedDRA - the Medical Dictionary for Regulatory Activities — lists medical terminology used to classify adverse events associated with the use of biopharmaceuticals and other medical products.)

All adverse events coded as infections were grouped into nine non-exclusive categories: nontuberculosis pneumonia, all respiratory infections, soft-tissue infections, gastrointestinal infections, urinary tract infections, gynecologic infections, viral infections, fungal infections and systemic sepsis. For participants who had more than one infection, the first occurrence within each category was used in the primary analyses.

Incident pneumonia was the main focus of the current study. The following MedDRA codes as reported by the study investigators were used to define pneumonia: bronchopneumonia (22 cases), pneumonia (441), Chlamydia pneumonia (2), Haemophilus pneumonia (1), Moraxella pneumonia (1), pneumoccocal pneumonia (1), atypical pneumonia (1), staphylococcal pneumonia (1), viral pneumonia (1) and bacterial pneumonia (7). We also performed an analysis of total pneumonia, in which recurrent events that occurred at least 90 days apart were considered as independent events.

To assess the severity of the pneumonia, we used the definition of "serious adverse event" as reported by the study investigators. For this analysis, an adverse event was considered serious if it was fatal, life-threatening, resulted in admission to hospital or prolongation of a hospital stay, required intervention to prevent permanent impairment or damage, or resulted in persistent or significant disability.

\section{Statistical analysis}

Analyses were done on an intention-to-treat basis. We used univariable Cox regression models to assess the rate ratio associated with rosuvastatin therapy and each category of infection. We used the Kaplan-Meier method to assess time to incident pneumonia.

Because cardiovascular events such as venous thromboembolism can result in an increased risk of infection (and because cardiovascular events were reduced by more than $40 \%$ in the rosuvastatin arm of the JUPITER trial ${ }^{13,14}$ ), we performed a sensitivity analysis in which patients were censored at the time of a cardiovascular event (nonfatal myocardial infarction, nonfatal stroke, unstable angina requiring admission to hospital, arterial revascularization, venous thromboembolism or confirmed death from a cardiovascular cause). This is a standard approach to account for competing risks. ${ }^{15}$

We investigated the association between rosuvastatin treatment and the occurrence of all pneumonias, including multiple events in some participants. For this analysis, we considered recurrent pneumonia as a separate event if it occurred at least 90 days after the first event. The overall incidence of pneumonia, including recurrent events, was calculated as the total number of events divided by the total person-time until participants had their closeout visit. ${ }^{16}$ We estimated the relative hazard of pneumonia, counting recurrent events in a proportional hazards model 
that used a robust variance estimate to account for possible overdispersion associated with multiple events per person. ${ }^{17}$

Following an approach recommended by Cox, ${ }^{18}$ we evaluated the proportional hazards assumption in our models by including an interaction term between age and log study time, with log time centred on the mean. In the presence of a nominal violation of this assumption $(p<0.05)$, we fitted separate proportional hazards models, as was done in the initial report from the trial. In addition, we examined possible heterogeneity in the effect of rosuvastatin on pneumonia across geographic regions through the use of a likelihood ratio test of interaction. For this analysis, we classified participants into five regions previously considered in the JUPITER trial: Canada and the United States; Central and South America; Europe; South Africa; and Israel. To investigate potential associations between risk factors and pneumonia, and whether control for these risk factors would influence the estimated treatment effect, we fitted a proportional hazards model with a robust variance estimate that counted all pneumonia events after treatment assignment and included as independent variables baseline age, sex, cigarette smoking, body mass index, presence of metabolic syndrome, total cholesterol and triglyceride levels, level of high-sensitivity Creactive protein and treatment assignment.

All $p$ values and 95\% confidence intervals (CIs) are two-sided.

\section{Role of the sponsor}

The JUPITER trial was initiated by the investigators and was supported financially by AstraZeneca. The sponsor collected the trial data and monitored the study sites but played no role in the conduct of the analyses or drafting of the manuscript.

\section{Results}

The characteristics of the participants at baseline were equally distributed in the rosuvastatin and placebo groups (Table 1). With regard to risk factors for pneumonia, $32 \%$ of the participants were 70 years or older at baseline, $37.6 \%$ had a body mass index of $30 \mathrm{~kg} / \mathrm{m}^{2}$ or greater, $41.3 \%$ had metabolic syndrome, and $16 \%$ were current smokers.

\section{Incident pneumonia}

Among the 17802 trial participants followed for a median of 1.9 years (maximum 5.0 years), incident pneumonia was reported as an adverse event in 214 participants assigned to the rosuva- statin group and in 257 assigned to the placebo group (hazard ratio [HR] 0.83, 95\% CI 0.691.00) (Table 2, Figure 1). This analysis included all first events of incident pneumonia, including those that occurred after a cardiovascular event. The number of participants with incident pneumonia classified as a serious adverse event did not differ significantly between the study groups (71 in the rosuvastatin group and 75 in the placebo group; HR $0.95,95 \%$ CI 0.68-1.31).

In the analyses restricted to events occurring before a cardiovascular event, pneumonia occurred in 203 participants given rosuvastatin and in 250 given placebo (HR $0.81,95 \% \mathrm{CI}$ 0.67-0.97) (Table 2, Figure 2).

In the analysis that included all instances of pneumonia during follow-up, including recurrences, there were 231 distinct pneumonia events in the rosuvastatin group (rate 1.19 per 100 person-years) and 285 in the placebo group (rate 1.47 per 100 person-years). The proportional hazards model accounting for recurrent events found an estimated relative hazard in the rosuvastatin group of 0.81 (95\% CI 0.67-0.98).

Table 1: Baseline characteristics of participants in the JUPITER trial, by study group

\begin{tabular}{|c|c|c|}
\hline \multirow[b]{2}{*}{ Characteristic } & \multicolumn{2}{|c|}{ Study group; no. (\%) of participants* } \\
\hline & $\begin{array}{l}\text { Rosuvastatin } \\
n=8901\end{array}$ & $\begin{array}{l}\text { Placebo } \\
n=8901\end{array}$ \\
\hline \multicolumn{3}{|l|}{ Age, yr } \\
\hline$<60$ & $1846(20.7)$ & $1843(20.7)$ \\
\hline $60-69$ & $4177(46.9)$ & $4241(47.6)$ \\
\hline$\geq 70$ & $2878(32.3)$ & $2817(31.6)$ \\
\hline Female sex & $3426(38.5)$ & 3375 (37.9) \\
\hline Current smoker & $1400(15.7)$ & $1420(16.0)$ \\
\hline Metabolic syndrome & $3652(41.0)$ & $3723(41.8)$ \\
\hline Body mass index, $\mathrm{kg} / \mathrm{m}^{2}$ & $n=8870$ & $n=8878$ \\
\hline$<25$ & $2040(23.0)$ & $2033(22.9)$ \\
\hline $25-<30$ & 3495 (39.4) & $3514(39.6)$ \\
\hline$\geq 30$ & $3338(37.6)$ & $3336(37.6)$ \\
\hline $\begin{array}{l}\text { High-sensitivity C-reactive protein } \\
\text { level, } † \mathrm{nmol} / \mathrm{L} \text {, median (IQR) }\end{array}$ & $40.0(26.7-67.6)$ & $41.0(26.7-68.6)$ \\
\hline \multicolumn{3}{|l|}{ Lipid level, mmol/L, median (IQR) } \\
\hline LDL cholesterol & $2.79(2.43-3.08)$ & $2.79(2.43-3.08)$ \\
\hline HDL cholesterol & $1.27(1.03-1.56)$ & $1.27(1.03-1.56)$ \\
\hline Triglycerides & $1.33(0.96-1.91)$ & $1.33(0.97-1.91)$ \\
\hline Total cholesterol & $4.81(4.34-5.17)$ & $4.78(4.37-5.15)$ \\
\hline \multicolumn{3}{|c|}{$\begin{array}{l}\text { Note: } \mathrm{HDL}=\text { high-density lipoprotein, IQR }=\text { interquartile range, JUPITER = Justification for } \\
\text { the Use of Statins in Prevention: an Intervention Trial Evaluating Rosuvastatin, LDL = low- } \\
\text { density lipoprotein. } \\
\text { *Unless stated otherwise. } \\
\text { tC-reactive protein levels were reported as averages based on levels from two visits before } \\
\text { the start of the trial (screening and randomization visits). }\end{array}$} \\
\hline
\end{tabular}


The estimated treatment effect was only nominally changed after we adjusted for age, sex, smoking, presence of metabolic syndrome, lipid levels and level of high-sensitivity C-reactive protein (HR 0.82, 95\% CI 0.68-0.99). Inclusion in this model of baseline risk factors found associations of older age and cigarette smoking with occurrence of pneumonia (each $p<0.001$ ), and weak associations of other risk factors (sex, body mass index, metabolic syndrome, triglyceride level and level of total cholesterol; each $p>0.05$ ), but left nearly unchanged the estimated treatment effect (relative hazard 0.82, 95\% CI 0.67-0.99).

Consideration of time to occurrence of pneumonia (Figures 1 and 2) suggested little effect of treatment in early follow-up and a larger effect later on. This suggestion of a time-varying effect was supported by a nominally significant test of the proportional hazards assumption $(p=0.04)$. Post-hoc analyses stratified by time found little treatment effect in the first two years of followup (362 events; HR 0.92, 95\% CI 0.75-1.14) but a greater effect after two years (109 events; HR $0.58,95 \%$ CI $0.39-0.85$ ).

Evaluation of possible heterogeneity in the effect of rosuvastatin by geographic region of participants found no evidence of such heterogeneity $(p=0.44)$.

\section{Other infections}

Incident infections other than pneumonia were diagnosed in 3760 participants in the rosuvastatin group and 3828 in the placebo group (HR $0.98,95 \%$ CI $0.94-1.03$ ). The event was classified as serious among 412 participants in the rosuvastatin group and 456 in the placebo group
(HR 0.90, 95\%CI 0.79-1.03). We observed no difference between the rosuvastatin and placebo groups for the following MedDRA categories: all respiratory infections, gastrointestinal infections, urinary tract infections, viral infections and systemic sepsis. In contrast, as observed for pneumonia, we observed reductions in risk associated with rosuvastatin in the categories of softtissue infections, gynecologic infections and fungal infections. None of the MedDRA categories of infection had a significantly higher incidence in the rosuvastatin group than in the placebo group (Table 2).

\section{Interpretation}

In the JUPITER trial, participants randomly assigned to receive rosuvastatin had a modest reduction in the incidence of pneumonia compared with participants assigned to the placebo group. This effect was unchanged in magnitude and remained statistically significant in analyses that adjusted for baseline determinants of pneumonia, in analyses that excluded pneumonia events occurring subsequent to a cardiovascular event and in analyses that included recurrent pneumonia events as well as first events.

Although a number of observational studies have suggested a protective effect of statin use on the incidence of pneumonia and other infections, ${ }^{2-8}$ we are not aware of any evidence from prior randomized trials that specifically evaluated this question. However, in a recent trial in which randomly selected patients admitted to hospital because of acute infection had their statin treatment substituted for placebo during their hospital stay, the odds ratio for progression to severe sep-

Table 2: Number, incidence (per 100 person-years) and hazard ratios for Infections reported as adverse events during follow-up among the 17802 trial participants

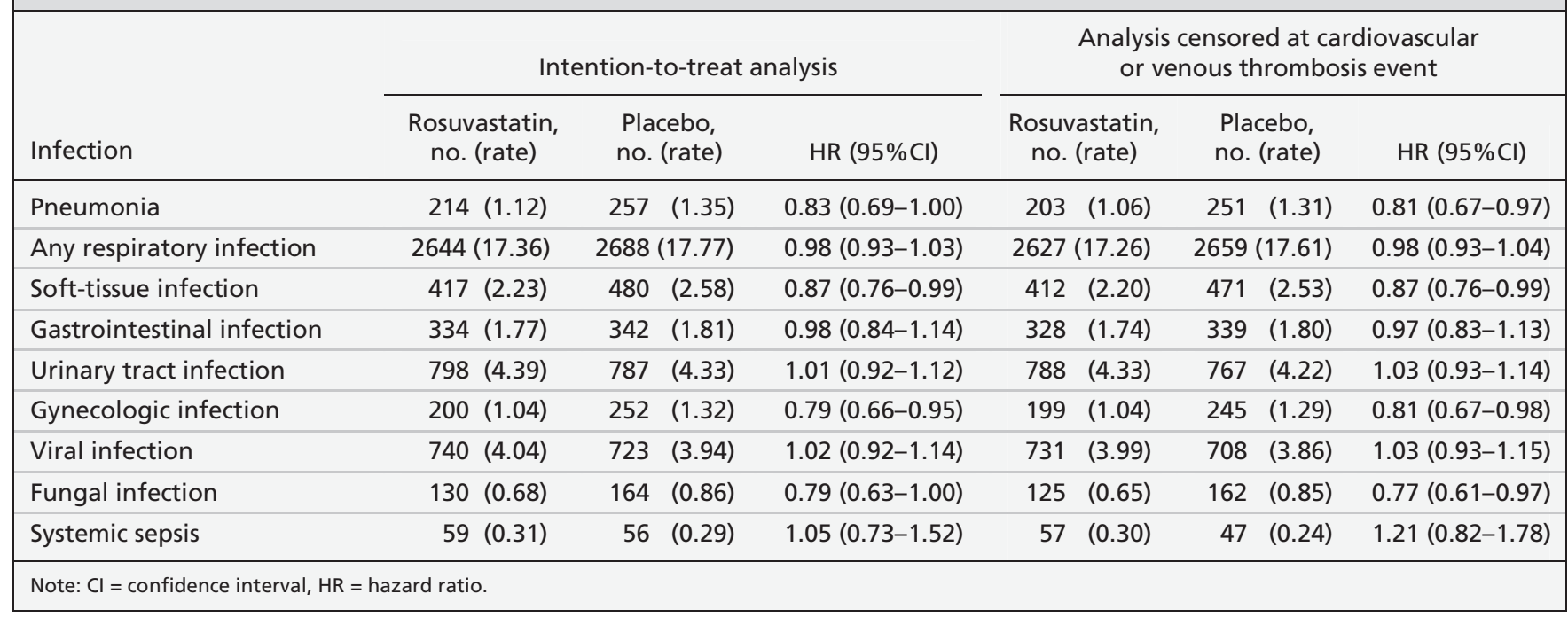


sis declined at a rate similar to that in the group whose statin treatment was not changed. ${ }^{19}$

Potential mechanisms that might support a protective effect of statins for infectious diseases include mild anti-inflammatory, antioxidant, immunomodulatory, anti-apoptotic and endothelial protection. Some laboratory data suggest that pretreatment with statins attenuates sepsis-induced shock; in a rat model, pretreatment with simvastatin decreased overproduction of nitric oxide and reverted the impaired vascular responsiveness induced by endotoxic shock. ${ }^{20}$ Similarly, in a randomized study, simvastatin treatment in patients admitted with infection was found to reduce levels of interleukin- 6 and tumour necrosis factor $\alpha .^{21}$

\section{Strengths and limitations}

The design of the JUPITER trial has several features that support the validity of our findings. Most importantly, treatment was assigned on a random basis. As such, the possibility was reduced that a decreased risk of pneumonia among rosuvastatin users was due to a "healthy cohort effect" or that rosuvastatin use was simply a surrogate for better general health habits, improved socioeconomic status or better access to care. Second, study investigators reported adverse events without knowing the participants' treatment assignments. Third, we performed analyses that excluded participants in whom pneumonia occurred after a cardiovascular event. By so doing, we were able to reduce greatly any bias that might accrue because of competing risks and that might lead to an apparent reduction in pneumonia or other infections simply as a result of differential cardiovascular protection.

Our study has limitations. The JUPITER trial was not designed to study a preventive effect for pneumonia, and the outcomes we used were not defined before the start of the trial. Rather, we relied on investigators' reports of adverse events, which were coded using standardized MedDRA criteria and not directly adjudicated. Thus, there may have been some misclassification and underreporting in our study. However, we focused our primary analysis on incident pneumonia, which we believe is less susceptible to misclassification and underreporting than mild upper respiratory infections or infections presumed to have a viral origin. Further, we know of no systematic reason that misclassification or underreporting would occur more often among participants given rosuvastatin than among those given placebo. As such, any random misclassification might have biased our point estimate of effect toward the null but would unlikely have led to a false-positive finding.
An additional limitation is that we did not have information on the patients' pneumococcal and influenza vaccination status. However, because of the randomized nature of the JUPITER trial, an imbalance on this basis is unlikely. Finally, the JUPITER trial was limited to healthy patients with low levels of LDL cholesterol and elevated levels of high-sensitivity Creactive protein, issues that may reduce generalizability of the findings.

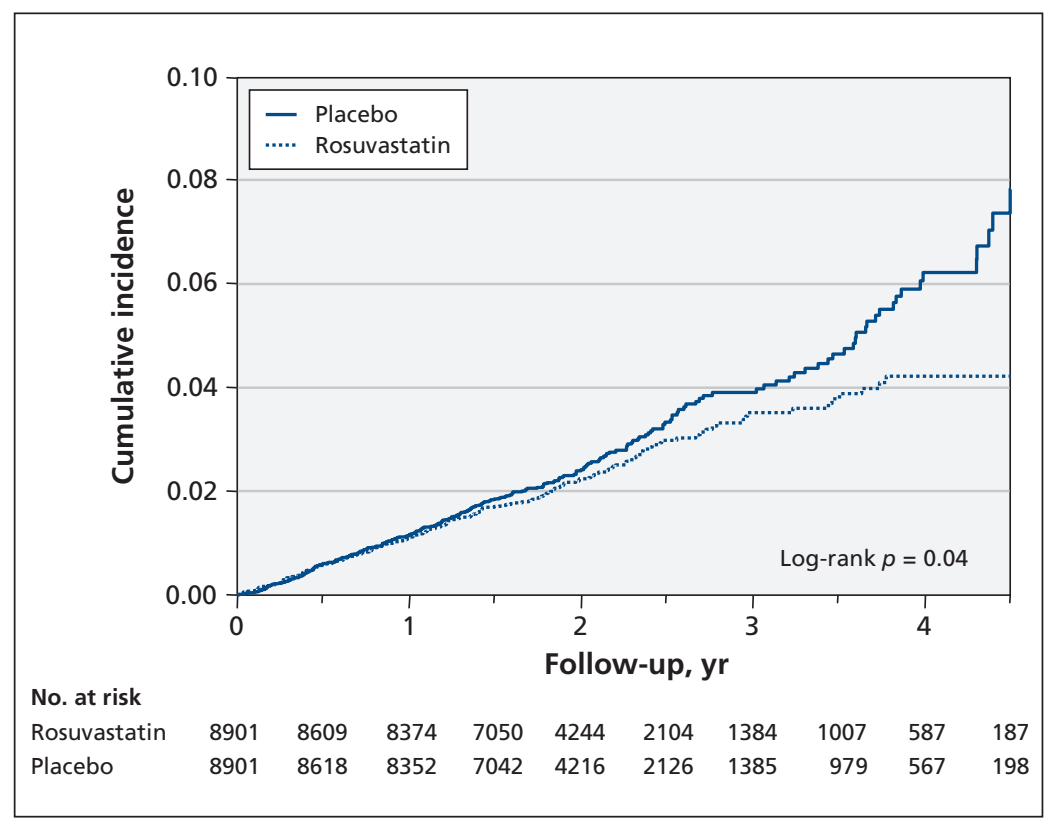

Figure 1: Kaplan-Meier estimates from intention-to-treat analysis showing cumulative incidence of first pneumonia among 17802 participants randomly assigned to receive either rosuvastatin or placebo.

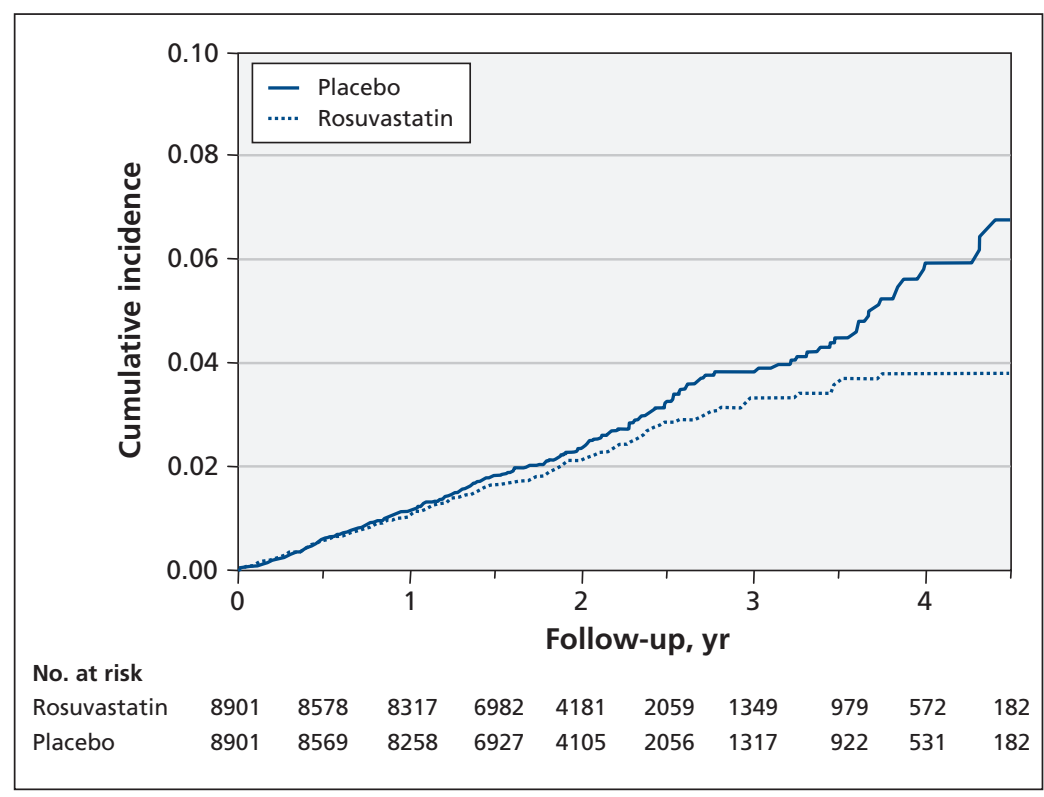

Figure 2: Kaplan-Meier estimates from intention-to-treat analysis showing cumulative incidence of first pneumonia adjusted for competing risk of cardiovascular events. Participants were censored at the time of a cardiovascular event. 


\section{Conclusion}

Data from the JUPITER trial support the hypothesis that statin treatment may modestly reduce the incidence of pneumonia. However, the absolute risk reduction observed in this primary prevention setting was small, and the effects on infection may be greater in other settings. As such, these data provide support for ongoing studies such as the Statins for Acutely Injured Lungs from Sepsis (SAILS) trial and emphasize the need for basic investigators to continue exploring novel mechanisms by which statin therapy appears to reduce the incidence of clinical events.

\section{References}

1. Baigent C, Keech A, Kearney PM, et al. Efficacy and safety of cholesterol-lowering treatment: prospective meta-analysis of data from 90056 participants in 14 randomised trials of statins. Lancet 2005;366:1267-78.

2. Almog Y, Novack V, Eisinger M, et al. The effect of statin therapy on infection-related mortality in patients with atherosclerotic diseases. Crit Care Med 2007;35:372-8.

3. Almog Y, Shefer A, Novack V, et al. Prior statin therapy is associated with a decreased rate of severe sepsis. Circulation 2004; 110:880-5.

4. Hackam DG, Mamdani M, Li P, et al. Statins and sepsis in patients with cardiovascular disease: a population-based cohort analysis. Lancet 2006;367:413-8.

5. Thomsen RW, Riis A, Kornum JB, et al. Preadmission use of statins and outcomes after hospitalization with pneumonia: population-based cohort study of 29,900 patients. Arch Intern Med 2008;168:2081-7.

6. Schlienger RG, Fedson DS, Jick SS, et al. Statins and the risk of pneumonia: a population-based, nested case-control study. Pharmacotherapy 2007;27:325-32.

7. van de Garde EM, Hak E, Souverein PC, et al. Statin treatment and reduced risk of pneumonia in patients with diabetes. Thorax 2006;61:957-61.

8. Myles PR, Hubbard RB, McKeever TM, et al. Risk of community-acquired pneumonia and the use of statins, ACE inhibitors and gastric acid suppressants: a population-based case-control study. Pharmacoepidemiol Drug Saf 2009;18:269-75.

9. Mortensen EM, Restrepo MI, Anzueto A, et al. The effect of prior statin use on 30-day mortality for patients hospitalized with community-acquired pneumonia. Respir Res 2005;6:82

10. Jain MK, Ridker PM. Anti-inflammatory effects of statins: clinical evidence and basic mechanisms. Nat Rev Drug Discov 2005; 4:977-87.

11. Majumdar SR, McAlister FA, Eurich DT, et al. Statins and outcomes in patients admitted to hospital with community-acquired pneumonia: population based prospective cohort study. BMJ 2006;333:999.

12. Brookhart MA, Patrick AR, Dormuth C, et al. Adherence to lipid-lowering therapy and the use of preventive health services: an investigation of the healthy user effect. Am J Epidemiol 2007; 166:348-54.

13. Ridker PM, Danielson E, Fonseca FA, et al. Rosuvastatin to prevent vascular events in men and women with elevated C-reactive protein. $N$ Engl J Med 2008;359:2195-207.

14. Glynn RJ, Danielson E, Fonseca FA, et al. A randomized trial of rosuvastatin in the prevention of venous thromboembolism. N Engl J Med 2009;360:1851-61.

15. Glynn RJ, Rosner B. Comparison of risk factors for the competing risks of coronary heart disease, stroke, and venous thromboembolism. Am J Epidemiol 2005;162:975-82.
16. Glynn RJ, Buring JE. Ways of measuring rates of recurrent events. BMJ 1996;312:364-7.

17. Wei LJ, Lin DY, Weissfeld L. Regression analysis of multivariate incomplete failure time data by modeling marginal distributions. J Am Stat Assoc 1989; 84:1065-73.

18. Cox D. Regression models and life tables. J R Stat Soc Series B Stat Methodol 1972;187-220.

19. Kruger PS, Harward ML, Jones MA, et al. Continuation of statin therapy in patients with presumed infection: a randomized controlled trial. Am J Respir Crit Care Med 2011;183:774-81.

20. Merx MW, Liehn EA, Graf J, et al. Statin treatment after onset of sepsis in a murine model improves survival. Circulation 2005; 112:117-24.

21. Novack V, Eisinger M, Frenkel A, et al. The effects of statin therapy on inflammatory cytokines in patients with bacterial infections: a randomized double-blind placebo controlled clinical trial. Intensive Care Med 2009;35:1255-60.

Competing interests: Paul Ridker was the principal investigator of the JUPITER trial, which was funded by AstraZeneca; he is a consultant to AstraZeneca and is listed as a co-inventor on patents held by the Brigham and Women's Hospital that relate to the use of inflammatory biomarkers in cardiovascular disease that have been licensed to Siemens and AstraZeneca; and he has received consultancy fees from Merck, ISIS, Vascular Biogenics and Genzyme, and a research grant from Novartis. Robert Glynn has received funding from AstraZeneca for the analysis of data from the JUPITER trial; he has received speaker fees from Merck and a research grant from Novartis. Atul Malhotra has received research grants from the National Institutes of Health, the American Heart Association, Philips, Sepracor and Cephalon; he has received consultancy fees from Philips, Pfizer, Merck, Apnex Medical, ApniCure, Ethicon, Medtronic, SHC Pharmaceuticals, SGS Pharmaceuticals and Galleon Pharmaceuticals. No competing interests for Victor Novack, Yaniv Almog and Jean MacFadyen.

Affiliations: From the Clinical Research Center (Novack) and the Medical Intensive Care Unit (Almog), Soroka University Medical Center, Beer-Sheva, Israel; the Harvard Clinical Research Institute (Novack), Boston, Mass.; and the Center for Cardiovascular Disease Prevention (MacFadyen, Glynn, Ridker), the Division of Cardiovascular Medicine (Ridker) and the Divisions of Sleep Medicine and of Pulmonary and Critical Care (Malhotra), Brigham and Women's Hospital, Harvard Medical School, Boston, Mass.

Contributors: Paul Ridker had full access to all of the data in the study and takes responsibility for the integrity of the data and the accuracy of the analyses. Victor Novack, Atul Malhotra, Yaniv Almog and Paul Ridker contributed to the study concept and design. Jean MacFadyen, Robert Glynn and Paul Ridker contributed to the acquisition of data. Victor Novack, Jean MacFadyen and Robert Glynn performed the statistical analyses. All of the authors analyzed and interpreted the data. Paul Ridker, Jean MacFadyen and Robert Glynn provided administrative, technical and material support. Victor Novack and Paul Ridker supervised the study. All of the authors contributed to the drafting of the manuscript, critically revised the manuscript for important intellectual content and approved the final version submitted for publication.

Funding: The JUPITER trial was initiated by the investigators and was supported financially by AstraZeneca. The sponsor collected the trial data and monitored the study sites but played no role in the conduct of the analyses or the preparation and review of the manuscript. 